\title{
Moving forward the in vivo diagnosis of the synucleinopathies
}

\author{
Dolores Vilas ${ }^{1}$ \\ Received: 21 January 2019 / Accepted: 23 January 2019 / Published online: 5 February 2019 \\ (c) Springer-Verlag GmbH Germany, part of Springer Nature 2019
}

Keywords Parkinson disease $\cdot$ Multiple system atrophy $\cdot$ Pure autonomic failure $\cdot$ Skin biopsy $\cdot$ Alpha-synuclein

\begin{abstract}
Abnormal aggregates of alpha-synuclein ( $\alpha$-syn) in the nervous system are the neuropathological hallmark of the synucleinopathies. Based on autopsy studies, we know that these abnormal deposits occur not only in the central nervous system in these disorders but also in the peripheral autonomic nervous system [1, 2]. The most common synucleinopathies are Parkinson disease (PD) and dementia with Lewy bodies (DLB); much less common synucleinopathies are multiple system atrophy and pure autonomic failure (PAF). The diagnosis of these diseases is currently still based on clinical criteria. A more accurate in vivo diagnosis is of major interest since it could lead to the early detection and treatment of patients affected by these diseases. However, reliable biomarkers are still lacking.
\end{abstract}

In the hope of identifying a diagnostic biomarker of synucleinopathies, researchers have been searching for peripheral $\alpha$-syn deposits in patients, initially in the olfactory epithelium and subsequently in the stomach, the intestines, and salivary glands.

In this issue of Clinical Autonomic Research, V. Donadio reviews the use of skin biopsies for the search of peripheral $\alpha$-syn deposits in patients with synucleinopathies [3]. In this article the author summarizes current progress in this area, reviewing different methodological aspects to facilitate the standardization of the method and eliminate differences between the various approaches used in different laboratories.

Skin innervation is easily accessible and contains a large number of autonomic nerve fibers. The peripheral sympathetic nerves have been proposed to be the earliest affected part of the nervous system in synucleinopathies. In vivo

Dolores Vilas

dvilas.germanstrias@gencat.cat

1 Movement Disorders Unit, Neurology Service, Hospital Universitari Germans Trias I Pujol, Badalona, Barcelona, Spain studies have reported on the presence of $\alpha$-syn aggregates in patients with PD and other synucleinopathies, with variable frequencies ranging from 0 to $100 \%$ [4-6]. This variable rate of detection of abnormal $\alpha$-syn aggregates could be, at least in part, due to the different methodological aspects of the different studies. In his paper, Donadio reviews some of these aspects, including the skin site, the number of biopsies performed, the method use for tissue fixation, the number of skin sections, the primary antibody used, and/or the immunohistochemical procedures. The majority of the reported studies reviewed used an indirect immunofluorescence technique that both utilizes primary antibodies against abnormal $\alpha$-syn aggregates and enables the localization of these aggregates; as such, this technique is the better option-at least at this point in time. Regarding the type of antibodies used, it would appear that those against $\alpha$-syn phosphorylated at Ser129 are the most efficacious in detecting $\alpha$-syn aggregates in the skin nerves. Donadio also found that the thickness of the skin sections varied widely among studies, which is mainly a reflection of differences in the underlying immunohistochemical technique used, leading to different probabilities of finding $\alpha$-syn aggregates.

The skin site and the number of biopsies performed are also variable. This aspect is of high relevance. A centripetal propagation of axonal $\alpha$-syn aggregates has been found to occur in the peripheral autonomic nervous system [7]. Moreover, samples from patients with PD and DLB without clinically significant autonomic failure present a patchy expression of phosphorylated $\alpha$-syn throughout all skin sites (i.e., proximal vs. distal), whereas samples from patients with marked autonomic failure (i.e., PAF, PD with orthostatic hypotension, DLB with orthostatic hypotension) show widespread deposits of phosphorylated $\alpha$-syn in nearly all analyzed proximal and distal skin samples [8]. Accordingly, the choice of skin site may be relevant when the aim is to search for abnormal $\alpha$-syn aggregates in synucleinopathies, especially in those conditions not associated with orthostatic 
hypotension. The number of skin biopsies may also be relevant when the distribution of $\alpha$-syn aggregates in skin nerves is irregular. In patients with PD and in DLB without orthostatic hypotension, the incidence of phosphorylated $\alpha$-syn in autonomic nerves differs between the proximal and distal skin sites, with the highest incidence observed in proximal sites [5, 9]. Similar findings were observed in samples from patients with idiopathic rapid eye movement sleep behavior disorder, a clinical condition that often precedes PD and DLB [10]. Proximal sites included the skin around the spine, i.e., close to autonomic ganglia, possibly suggesting that misfolded $\alpha$-syn comes from ganglia and spreads first to the closest autonomic skin terminals. This mechanism could explain why in some studies abnormal $\alpha$-syn aggregates in distal regions, such as the distal leg, were not found. A spine gradient, from proximal to distal, with a higher load of abnormal $\alpha$-syn deposits in the skin of the paravertebral cervical and the thoracic areas, has also been reported [10], although other studies did not confirm these findings. Available data support the hypothesis that the skin around the spine is the optimal site to search for phosphorylated $\alpha$-syn in skin nerves in synucleinopathies, with the cervical area being the site of choice. However, the optimal number of skin samples and sections needed to detect phosphorylated $\alpha$-syn in skin nerves remains to be defined. Finally, criteria for considering a biopsy positive or negative for $\alpha$-syn are not uniform among laboratories, making comparisons of results difficult.

Skin biopsy is a promising diagnostic tool for synucleinopathies because it is a low-cost and easy-to-perform procedure that causes only minor discomfort to the patient. However, standardization of these procedures is desirable to facilitate the large-scale automation of this technique. Obtaining a skin sample is a technically challenging process that cannot be done in a routine laboratory setting, at least not at this time. The search for peripheral $\alpha$-syn aggregates in synucleinopathies is still ongoing, and tissues other than the skin, such as the submandibular or the salivary glands, need further investigation.

\section{Compliance with ethical standards}

Conflict of interest The author declares that there is no conflict of interest.

\section{References}

1. Beach TG, Adler CH, Sue LI et al (2010) Multi-organ distribution of phosphorylated alpha-synucleinhistopathology in subjects with Lewy body disorders. Acta Neuropathol 119(6):689-702

2. Gelpi E, Navarro-Otano J, Tolosa E et al (2014) Multiple organ involvement by alpha-synuclein pathology in Lewy body disorders. Mov Disord 29(8):1010-1018

3. Vincenzo D (2018) Skin nerve $\alpha$-synuclein deposits in Parkinson's disease and other synucleinopathies: a review. Clin Auton Res. https://doi.org/10.1007/s10286-018-0581-4

4. Wang N, Gibbons CH, Lafo J, Freeman R (2013) $\alpha$-Synuclein in cutaneous autonomic nerves. Neurology. 81(18):1604-1610

5. Navarro-Otano J, Casanova-Mollà J, Morales M, Valls-Solé J, Tolosa E (2015) Cutaneous autonomic denervation in Parkinson's disease. J Neural Transm (Vienna). 122(8):1149-1155

6. Zange L, Noack C, Hahn K, Stenzel W, Lipp A (2015) Phosphorylated $\alpha$-synuclein in skin nerve fibres differentiates Parkinson's disease from multiple system atrophy. Brain 138(Pt 8):2310-2321

7. Orimo S, Uchihara T, Nakamura A et al (2008) Axonal alphasynuclein aggregates herald centripetal degeneration of cardiac sympathetic nerve in Parkinson's disease. Brain 131(Pt 3):642-650

8. Donadio V, Incensi A, Del Sorbo F et al (2018) Skin nerve phosphorylated $\alpha$-synuclein deposits in Parkinson disease with orthostatic hypotension. J Neuropathol Exp Neurol 77(10):942-949

9. Donadio V, Incensi A, Rizzo G et al (2017) A new potential biomarker for dementia with Lewy bodies: skin nerve $\alpha$-synuclein deposits. Neurology. 89(4):318-326

10. Antelmi E, Donadio V, Incensi A, Plazzi G, Liguori R (2017) Skin nerve phosphorylated $\alpha$-synuclein deposits in idiopathic REM sleep behavior disorder. Neurology 88(22):2128-2131 